\title{
Fuzzy Modeling and Control for a Class of Inverted Pendulum System
}

\author{
Liang Zhang, ${ }^{1}$ Xiaoheng Chang, ${ }^{1}$ and Hamid Reza Karimi ${ }^{2}$ \\ ${ }^{1}$ College of Engineering, Bohai University, Jinzhou 121013, China \\ ${ }^{2}$ Department of Engineering, Faculty of Engineering and Science, The University of Agder, 4898 Grimstad, Norway \\ Correspondence should be addressed to Liang Zhang; md18638@126.com
}

Received 14 December 2013; Accepted 24 December 2013; Published 29 January 2014

Academic Editor: Ming Liu

Copyright (c) 2014 Liang Zhang et al. This is an open access article distributed under the Creative Commons Attribution License, which permits unrestricted use, distribution, and reproduction in any medium, provided the original work is properly cited.

\begin{abstract}
Focusing on the issue of nonlinear stability control system about the single-stage inverted pendulum, the T-S fuzzy model is employed. Firstly, linear approximation method would be applied into fuzzy model for the single-stage inverted pendulum. At the same time, for some nonlinear terms which could not be dealt with via linear approximation method, this paper will adopt fan range method into fuzzy model. After the T-S fuzzy model, the PDC technology is utilized to design the fuzzy controller secondly. Numerical simulation results, obtained by Matlab, demonstrate the well-controlled effectiveness based on the proposed method for the model of T-S fuzzy system and fuzzy controller.
\end{abstract}

\section{Introduction}

Traditional control theory has perfect control ability for explicitly controlled system, however, which is a little weak to describe too complex or difficult system accurately. Therefore, many researchers seek ways to resolve this problem; those researchers have also focused on fuzzy mathematics and applied it to control problems. Zadeh [1] created fuzzy mathematics on an uncertainty system of control which is great contribution. Since the 70s, some practical controllers appear in succession, so that we have a big step forward in the control field. A number of control design approaches using adaptive control [2-4], sliding mode control [5, 6], $H_{\infty}$ [7-9], optimal control [10-12], control based data driven [10, 13-15], and fuzzy control $[16,17]$. The inverted pendulum system is controlled by the method of fuzzy control and realizes steady control. The inverted pendulum is a typical automatic control in the field of controlled object [18], which is multivariable and nonlinear and strong coupling characteristics, and so on. The inverted pendulum system reveals a natural unstable object, which can accomplish the stability and good performance by the control methods.

For the stability control of inverted pendulum system, the establishment of the model takes an important role. T$S$ fuzzy control [19] is the most popular one of the most promising methods based on modeling of fuzzy control research platform. At present, the T-S fuzzy control is one of the methods for nonlinear system control research [20], which is very popular. Based on T-S fuzzy model of inverted pendulum system modeling and control have a certain research. For inverted pendulum system based on T-S fuzzy mode, there are two methods [21]: the first one is the fan of nonlinear method. Although this method has high precision in describing the nonlinear system, it obtains many fuzzy rules. Thus it brings to the controller design difficulty, especially for the nonlinear term system. The second one is linear approximation modeling method, the method at the expense of the modeling accuracy and less number of rules of T-S fuzzy model. Since the second method can obtain a simple T$S$ fuzzy model, so in the inverted pendulum system modeling it is widely applied, but there is a very important problem, which is that if for one type of inverted pendulum system it contains the approximate method to deal with the nonlinear term, then the fuzzy modeling becomes the key to study.

Based on the above analysis and discussion, this thesis will carry the fuzzy modeling and control on inverted pendulum system of complex nonlinear term. For this point, sector nonlinear and linear approximation method will be adopted in the T-S fuzzy modeling of some inverted pendulums and the design of fuzzy controller. The fuzzy modeling and control 
method can achieve the stability control of the single inverted pendulum system through the simulation.

\section{Fuzzy Modeling for the Inverted Pendulum System}

Assume that the car's quality is $M$, the pendulum's quality is $m$, the pendulum's length is $l$, the pendulum's angle is $\theta$ at an instant (the angle between the pendulum rod and the vertical direction), the initial displacement is $x, g=9.8 \mathrm{~m} / \mathrm{s}^{2}$ is the gravity constant, the level for control is forced acting on the car is $F, a=1 /(m+M)$, and the inverted pendulum's state space is as follows:

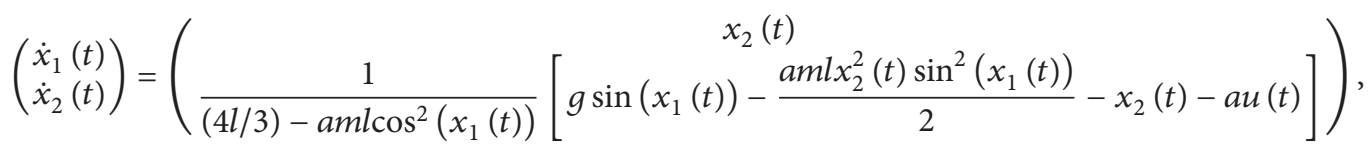

where $\theta$ is $x_{1}(t), \dot{\theta}$ is $x_{2}(t), F=u(t)$, and $x_{1}(t) \in(0, \pm \pi / 2)$, $x_{2}(t) \in[-\alpha, \alpha]$.

When $x_{1}(t)= \pm \pi / 2$, the system is uncontrollable, so we take $x_{1}(t) \in\left[-88^{\circ}, 88^{\circ}\right]$ as the range. For this inverted pendulum system, T-S fuzzy model can be considered as follows:

$$
\begin{gathered}
R^{i}: \text { if } x_{1}(t) \text { is } M_{1}^{i}, \ldots x_{n}(t) \text { is } M_{n}^{i} \\
\text { then } \dot{x}(t)=A_{i} x(t)+B_{i} u(t),
\end{gathered}
$$

where $x(t)=\left[\begin{array}{llll}x_{1}(t) & x_{2}(t) & \cdots & x_{n}(t)\end{array}\right]^{T} \in R^{n}$ is the state variables for the fuzzy system, $M_{k}^{i}$ is the fuzzy sets and where $k=$ $1,2, \ldots, n, i=1,2, \ldots, r$, the input vector is $u(t) \in R^{m}, A_{i} \in$ $R^{n \times n}, B_{i} \in R^{n \times m}$ are coefficient matrix for the system. The number of fuzzy rules for the system is $r$.

The total fuzzy control system is as follows:

$$
\dot{x}(t)=\frac{\sum_{i=1}^{r} \omega_{i}(x(t))\left(A_{i} x(t)+B_{i} u(t)\right)}{\sum_{i=1}^{r} \omega_{i}(x(t))},
$$

where $\omega_{i}(x(t))=\prod_{k=1}^{n} M_{k}^{i}\left(x_{k}(t)\right)$, and $M_{k}^{i}\left(x_{k}(t)\right)$ is denotes the membership degree, and where $x_{k}(t)$ for $M_{k}^{i}$. The $h_{i}(x(t))=\omega_{i}(x(t)) / \sum_{j=1}^{r} \omega_{j}(x(t))$ and (2) will be as follows:

$$
\dot{x}(t)=\sum_{i=1}^{r} h_{i}(x(t))\left(A_{i} x(t)+B_{i} u(t)\right)
$$

where $h_{i}(x(t)) \geq 0$ and $\sum_{i=1}^{r} h_{i}(x(t))=1$.

There is an important nonlinear term in this inverted pendulum system, in other words $x_{2}^{2}(t) \sin ^{2}\left(x_{1}(t)\right)$, which should be paid more attention. The nonlinear term cannot be conducted through the linear approximation method on this inverted pendulum system. Thus, the thesis will combine the linear approximation method with the fan of nonlinear method to establish the fuzzy model. The process is as follows.

(1) If $x_{1}(t)$ is about 0 , through approximate treatment the system with the linear approximation method, the fuzzy model of system can be obtained as follows: then

$$
\begin{aligned}
\left(\begin{array}{c}
\dot{x}_{1}(t) \\
\dot{x}_{2}(t)
\end{array}\right)= & \left(\begin{array}{cc}
0 & 1 \\
\frac{g}{(4 l / 3)-a m l} & \frac{-1}{(4 l / 3)-a m l}
\end{array}\right)\left(\begin{array}{l}
x_{1}(t) \\
x_{2}(t)
\end{array}\right) \\
& +\left(\begin{array}{c}
0 \\
\frac{-a}{(4 l / 3)-a m l}
\end{array}\right) u(t) \\
\left(\begin{array}{c}
\dot{x}_{1}(t) \\
\dot{x}_{2}(t)
\end{array}\right)= & \left(\begin{array}{cc}
0 & 1 \\
\frac{3 g}{4 l-3 a m l} & \frac{-3}{4 l-3 a m l}
\end{array}\right)\left(\begin{array}{l}
x_{1}(t) \\
x_{2}(t)
\end{array}\right) \\
& +\left(\begin{array}{c}
0 \\
\frac{-3 a}{4 l-3 a m l}
\end{array}\right) u(t) .
\end{aligned}
$$

(2) If $x_{1}(t)$ is about $\pm \pi / 2$, and consider the fan of nonlinear method, and $z(t)=x_{2}(t) \sin ^{2}\left(x_{1}(t)\right)$,

then

$\max _{x_{1}(t), x_{2}(t)} z(t)=x_{2}(t) \sin ^{2}\left(x_{1}(t)\right) \equiv c_{1}=0.2595$.

Then

$$
\min _{x_{1}(t), x_{2}(t)} z(t)=x_{2}(t) \sin ^{2}\left(x_{1}(t)\right) \equiv c_{2}=-0.2595 .
$$

(1) If $z(t)$ is $c_{1}$, through the linear approximation method and the fan of nonlinear method to approximate treatment, the fuzzy model of system can be obtained as follows:

$$
\begin{aligned}
& \left(\begin{array}{c}
\dot{x}_{1}(t) \\
\dot{x}_{2}(t)
\end{array}\right)=\left(\begin{array}{cc}
0 & 1 \\
\frac{g(2 / \pi)}{4 l / 3} & -\left(\frac{1}{4 l / 3} \frac{a m l c_{1}}{2}+\frac{1}{4 l / 3}\right)
\end{array}\right)\left(\begin{array}{l}
x_{1}(t) \\
x_{2}(t)
\end{array}\right) \\
& +\left(\begin{array}{c}
0 \\
\frac{-a}{4 l / 3}
\end{array}\right) u(t)
\end{aligned}
$$$$
\left.\left(\begin{array}{c}
\dot{x}_{1}(t) \\
\dot{x}_{2}(t)
\end{array}\right)=\left(\begin{array}{cc}
0 & 1 \\
\frac{3 g}{2 \pi l} & -\left(\frac{3 a m c_{1}}{8}+\frac{3}{4 l}\right.
\end{array}\right)\right)\left(\begin{array}{c}
x_{1}(t) \\
x_{2}(t)
\end{array}\right)+\left(\begin{array}{c}
0 \\
\frac{-3 a}{4 l}
\end{array}\right) u(t) .
$$ 


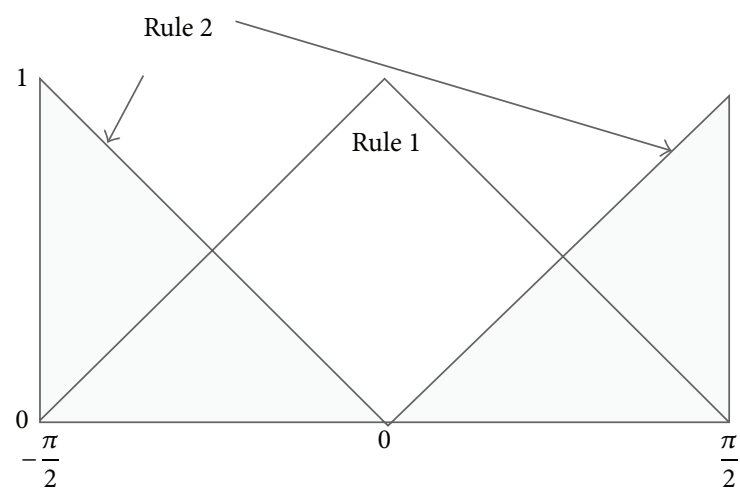

FIGURE 1: Membership functions of two-rule model.

(2) If $z(t)$ is $c_{2}$, through the linear approximation method and the fan of nonlinear method to approximate treatment, the fuzzy model of system can be obtained as follows:

$$
\begin{aligned}
\left(\begin{array}{c}
\dot{x}_{1}(t) \\
\dot{x}_{2}(t)
\end{array}\right)= & \left(\frac{g(2 / \pi)}{4 l / 3}-\left(\frac{1}{4 l / 3} \frac{a m l c_{2}}{2}+\frac{1}{4 l / 3}\right)\right)\left(\begin{array}{l}
x_{1}(t) \\
x_{2}(t)
\end{array}\right) \\
& +\left(\begin{array}{c}
0 \\
\frac{-a}{4 l / 3}
\end{array}\right) u(t),
\end{aligned}
$$$$
\left(\begin{array}{c}
\dot{x}_{1}(t) \\
\dot{x}_{2}(t)
\end{array}\right)=\left(\begin{array}{cc}
0 & 1 \\
\frac{3 g}{2 \pi l} & -\left(\frac{3 a m c_{2}}{8}+\frac{3}{4 l}\right)
\end{array}\right)\left(\begin{array}{l}
x_{1}(t) \\
x_{2}(t)
\end{array}\right)+\left(\begin{array}{c}
0 \\
\frac{-3 a}{4 l}
\end{array}\right) u(t) .
$$

Here define membership functions. For the part of linear approximation, the membership function is shown as Figure 1.

Rule 1: Consider

$$
H_{1}(t)=\left\{\begin{array}{ll}
\frac{2}{\pi} x_{1}(t)+1, & \left(-\frac{\pi}{2} \leq x_{1}(t) \leq 0\right) \\
-\frac{2}{\pi} x_{1}(t)+1, & \left(0<x_{1}(t) \leq \frac{\pi}{2}\right)
\end{array} .\right.
$$

Rule 2: Consider

$$
H_{2}(t)=1-H_{1}(t)
$$

Figure 2 is the membership function for the fan of nonlinear method. $z(t)$ can be rewritten as $z(t)=\sum_{i=1}^{2} E_{i}(z(t)) c_{i}$, where $E_{1}(z(t))=\left(z(t)-c_{2}\right) /\left(c_{1}-c_{2}\right)$ and $E_{2}(z(t))=\left(c_{1}-z(t)\right) /\left(c_{1}-c_{2}\right)$. The membership functions $E_{1}(z(t))$ and $E_{2}(z(t))$ will meet the equation $E_{1}(z(t))+E_{2}(z(t))=1$.

In conclusion, the finally fuzzy model for the system will be shown as follows.

Rule 1: if $x_{1}(t)$ tends to 0 , then $\dot{x}(t)=A_{1} x(t)+B_{1} u(t)$.

Rule 2: if $x_{1}(t)$ tends to $\pm(\pi / 2)\left(\left|x_{1}(t)\right|<\pi / 2\right)$ and $z(t)$ takes the maximum value, then $\dot{x}(t)=A_{2} x(t)+$ $B_{2} u(t)$.

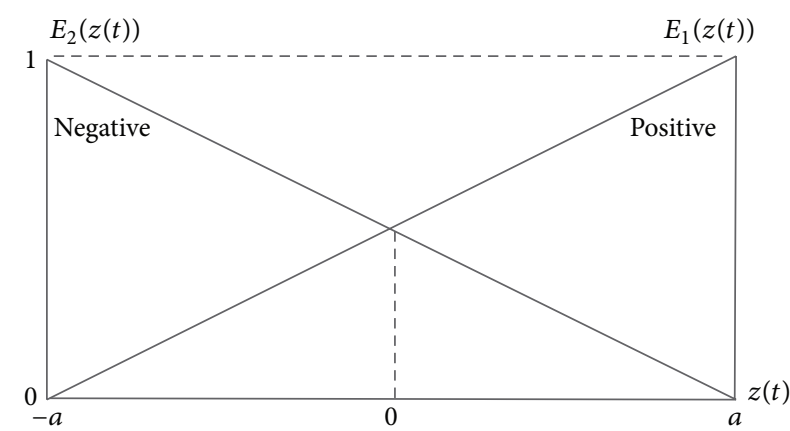

Figure 2: Membership function for the fan of nonlinear method.

TABLE 1: Function parameters.

\begin{tabular}{llc}
\hline Parameter & Function & Value \\
\hline$M$ & The mass of the cart & $1.096 \mathrm{~kg}$ \\
$m$ & The mass of the pendulum & $0.109 \mathrm{~kg}$ \\
$l$ & The length of the pendulum & $0.25 \mathrm{~m}$ \\
$\theta$ & The angle of the pendulum from & \\
$F$ & the vertical \\
\hline
\end{tabular}

Rule 3: if $x_{1}(t)$ tends to $\pm(\pi / 2)\left(\left|x_{1}(t)\right|<\pi / 2\right)$ and $z(t)$ takes the minimum value, then $\dot{x}(t)=A_{3} x(t)+$ $B_{3} u(t)$. Table 1 .

The function and value of every parameter are shown in

All the parameters defined in Table 1 are taken to account, then the system coefficient matrix can be obtained as follow

$$
\begin{aligned}
A_{1} & =\left(\begin{array}{cc}
0 & 1 \\
\frac{3 g}{4 l-3 a m l} & \frac{-3}{4 l-3 a m l}
\end{array}\right)=\left(\begin{array}{cc}
0 & 1 \\
31.5397 & -3.2183
\end{array}\right) \\
B_{1} & =\left(\begin{array}{c}
0 \\
\frac{-3 a}{4 l-3 a m l}
\end{array}\right)=\left(\begin{array}{c}
0 \\
-2.6708
\end{array}\right) \\
A_{2} & =\left(\begin{array}{c}
0 \\
\frac{3 g}{2 \pi l}-\left(\frac{3 a m c_{1}}{8}+\frac{3}{4 l}\right)
\end{array}\right)=\left(\begin{array}{cc}
0 & 1 \\
18.7166 & -3.0088
\end{array}\right) \\
B_{2} & =\left(\begin{array}{c}
0 \\
\frac{-3 a}{4 l}
\end{array}\right)=\left(\begin{array}{c}
0 \\
-2.4896
\end{array}\right) \\
A_{3} & \left.=\left(\begin{array}{c}
0 \\
\frac{g(2 / \pi)}{4 l / 3}-\left(\frac{1}{4 l / 3} \frac{a m l c_{2}}{2}+\frac{1}{4 l / 3}\right.
\end{array}\right)\right) \\
& =\left(\begin{array}{c}
0 \\
18.7166-2.9912
\end{array}\right) \\
B_{3} & =\left(\begin{array}{c}
0 \\
\frac{-a}{4 l / 3}
\end{array}\right)=\left(\begin{array}{c}
0 \\
-2.4896
\end{array}\right) .
\end{aligned}
$$




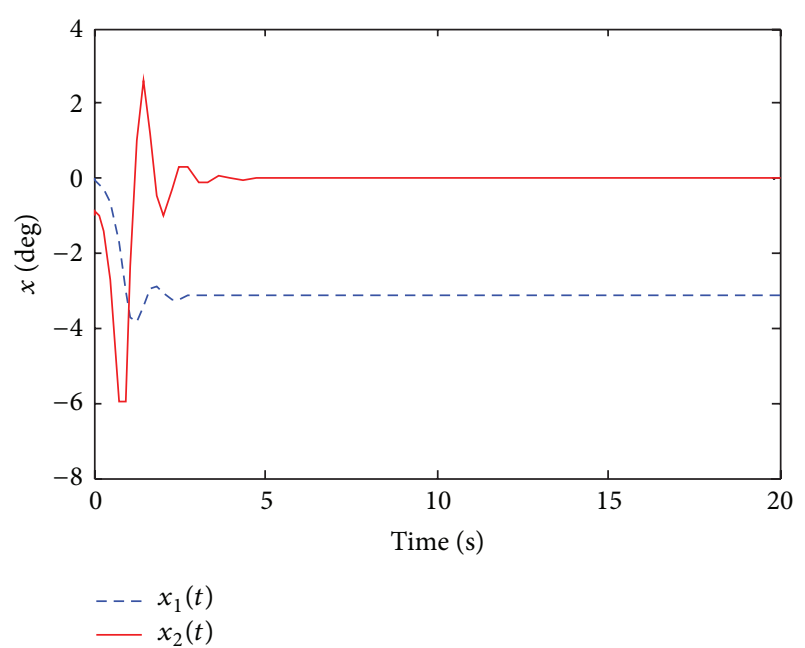

FIGURE 3: Simulation result without the controller.

The model rules can be represented as follows:

$$
\begin{gathered}
h_{1}(t)=H_{1}(t), \\
h_{2}(t)=H_{2}(t) \times E_{1}(z(t))=H_{2}(t) \times\left[\frac{z(t)-c_{2}}{c_{1}-c_{2}}\right], \\
h_{3}(t)=H_{2}(t) \times E_{2}(z(t))=H_{2}(t) \times\left[\frac{c_{1}-z(t)}{c_{1}-c_{2}}\right] .
\end{gathered}
$$

For the T-S model of the control object, a parallel distributed compensation control scheme (PDC) is employed. And the regulations are described as follow:

$$
\begin{aligned}
& R^{i}: \text { if } x_{1}(t) \text { is } M_{1}^{i}, \ldots x_{n}(t) \text { is } M_{n}^{i} \\
& \text { then } u(t)=K_{i} x(t) .
\end{aligned}
$$

Here the fuzzy controller and the fuzzy system adopt the same fuzzy rule. The overall model for the fuzzy controller is as follows:

$$
u(t)=\sum_{i=1}^{r} h_{i}(x(t)) K_{i} x(t)
$$

The closed control system can be obtained by combining (2) and (4):

$$
\dot{x}=\sum_{i=1}^{r} \sum_{j=1}^{r} h_{i} h_{j}\left(A_{i}+B_{i} K_{j}\right) x
$$

\section{Based on Linear Matrix Inequalities (LMI) and the Matlab Simulation}

Without the controller, the simulation output curves of the angular velocity and angular acceleration are shown in Figure 3.

Figure 3 shows that the inverted pendulum system is unstable without the controller.
In the following, by using the linear matrix inequalities technique [23], the fuzzy controller is designed.

Let us define the Lyapunov function as $(x(t))=$ $x^{T}(t) P x(t), P>0$, then the stable criterion of the system for (16) is as follows:

$$
\sum_{i=1}^{r} \sum_{j=1}^{r} h_{i} h_{j}\left(A_{i}+B_{i} K_{j}\right)^{T} P+P \sum_{i=1}^{r} \sum_{j=1}^{r} h_{i} h_{j}\left(A_{i}+B_{i} K_{j}\right)<0 .
$$

Define $Q=P^{-1}$; we can obtain (18) by multiplying $Q$ on both sides contemporary:

$$
Q \sum_{i=1}^{r} \sum_{j=1}^{r} h_{i} h_{j}\left(A_{i}+B_{i} K_{j}\right)^{T}+\sum_{i=1}^{r} \sum_{j=1}^{r} h_{i} h_{j}\left(A_{i}+B_{i} K_{j}\right) Q<0 .
$$

After defining $K_{j} Q=N_{j}$, the system stability discriminant conditions can be obtained. And this will guarantee a positive definite matrix $Q$ and matrix $N_{j}$ can be searched, then the following matrix inequality can be established:

$$
\begin{gathered}
\mathrm{Q} A_{i}^{T}+N_{i}^{T} B_{i}^{T}+A_{i} Q+B_{i} N_{i}<0, \\
Q A_{i}^{T}+N_{j}^{T} B_{i}^{T}+A_{i} Q+B_{i} N_{j}+Q A_{j}^{T}+N_{i}^{T} B_{j}^{T}+A_{j} Q+B_{j} N_{i}<0, \\
i=1,2, \ldots, r, i<j,
\end{gathered}
$$

where the stable controller will be obtained from (20):

$$
K_{j}=N_{j} Q^{-1}
$$

Through solving (19) by linear matrix inequality with LMI of Matlab [24], we can obtain

$$
\begin{aligned}
Q & =\left[\begin{array}{cc}
1.1127 & -0.4874 \\
-0.4874 & 1.9975
\end{array}\right], \\
N_{1} & =\left[\begin{array}{ll}
14.6725 & -7.9846
\end{array}\right], \\
N_{2} & =\left[\begin{array}{ll}
9.7868 & -5.9077
\end{array}\right], \\
N_{3} & =\left[\begin{array}{ll}
9.7836 & -5.8945
\end{array}\right] .
\end{aligned}
$$

Moreover, taking advantage of (20), the fuzzy controller gain will be obtained and shown as follows:

$$
\begin{aligned}
& K_{1}=\left[\begin{array}{ll}
12.8038 & -0.8733
\end{array}\right], \\
& K_{2}=\left[\begin{array}{ll}
8.3975 & -0.9086
\end{array}\right], \\
& K_{3}=\left[\begin{array}{ll}
8.3975 & -0.9020
\end{array}\right] .
\end{aligned}
$$

Put the controller gain into (16); design the simulation program in the simulink environment. Here the initial value $x(0)=[-0.01-0.1]$ is selected. The results of the fuzzy control simulation of the level single inverted pendulum system are shown in Figures 4, 5, and 6.

Simulation results show that the system responses converge to the equilibrium point, which indicates that the design of the controller is stable. 


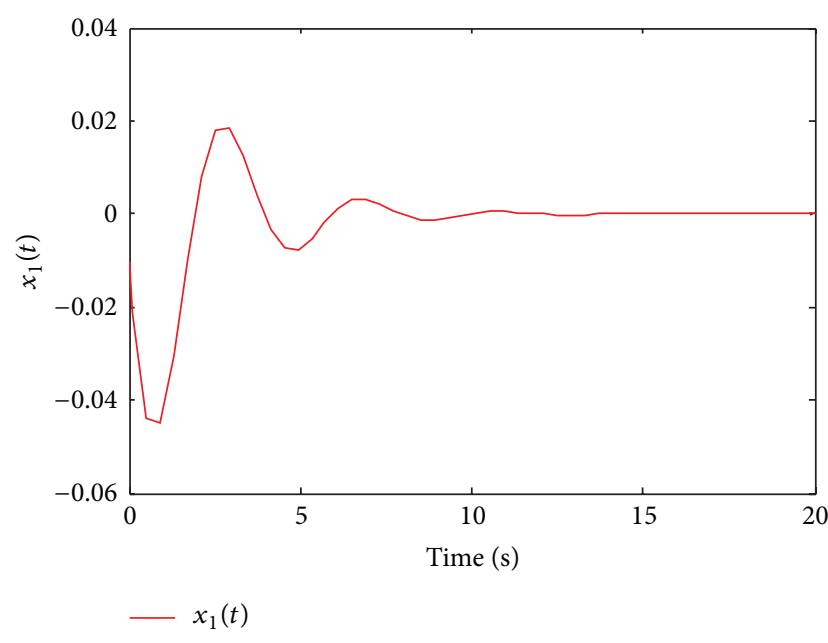

FIGURE 4: Simulation result of the angle.

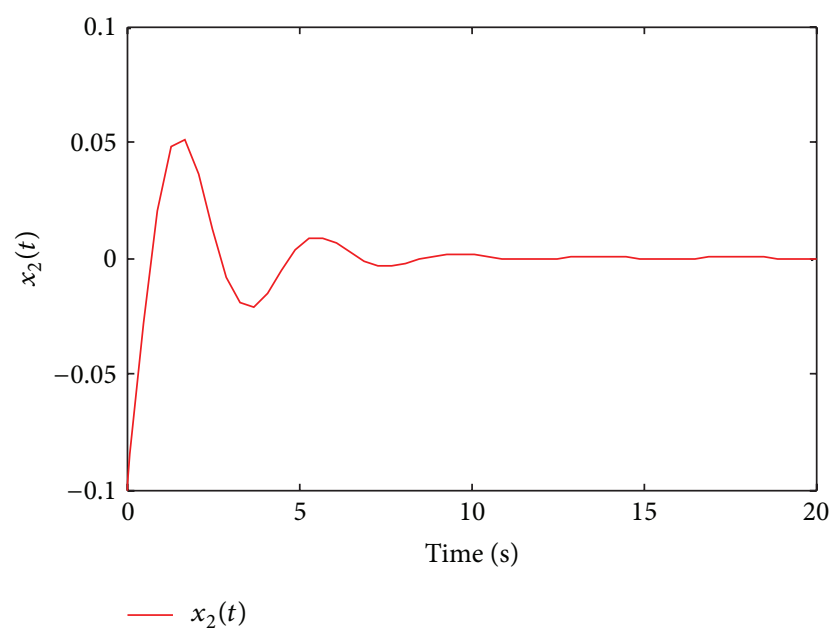

FIGURE 5: Simulation result of the angular velocity.

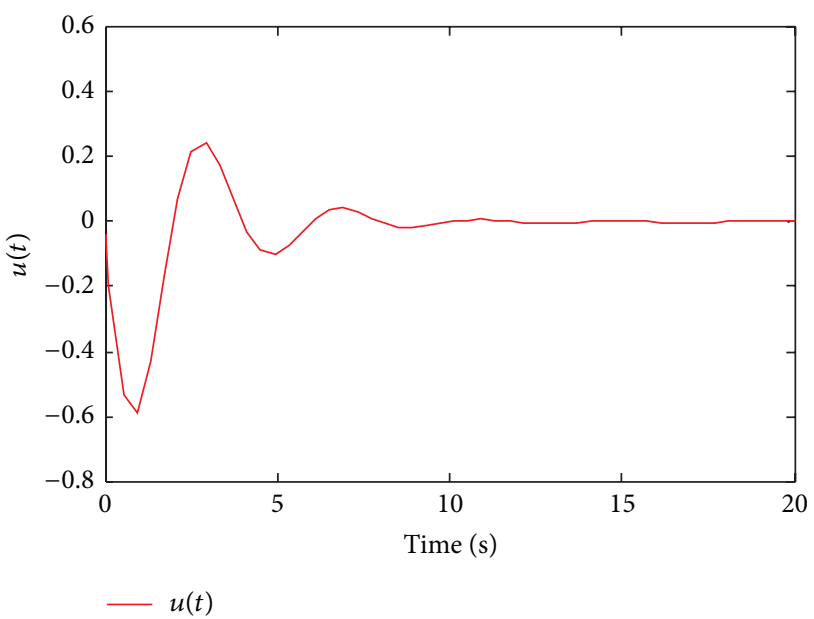

FIGURE 6: Simulation result of the controller.

\section{Conclusion}

This thesis takes a class of an inverted pendulum system as the research object. The system fuzzy model was established by the methods that combining with the linearization approximation processing and fan-shaped interval, and then the fuzzy controller was designed. Matlab-Simulink software toolbox was employed to be on computer simulation. The results show that it achieved a stable control of the single-stage inverted pendulum system through fuzzy control method on the basis of this fuzzy model. This model has the advantages of less fuzzy rules, high precision, and simple structure. The research results can provide an effective way for the subsequent instability in other nonlinear system modeling and fuzzy control.

\section{Conflict of Interests}

The authors declare that there is no conflict of interests regarding the publication of this paper.

\section{Acknowledgments}

This work is supported by the National Nature Science Foundation of China under Grant (Project no. 61104071), by the Program for Liaoning Excellent Talents in University, China, under Grant (Project no. LJQ2012095), by the Open Program of the Key Laboratory of Manufacturing Industrial Integrated Automation, Shenyang University, China, under Grant (Project no. 1120211415), and Natural Science Foundation of Liaoning, China (Project no. 2013020044). The authors highly appreciate the above financial supports.

\section{References}

[1] L. A. Zadeh, "Fuzzy sets," Information and Computation, vol. 8, pp. 338-353, 1965.

[2] B. Xiao, Q. Hu, and Y. Zhang, "Adaptive sliding mode fault tolerant attitude tracking control for flexible spacecraft under actuator saturation," IEEE Transactions on Control Systems Technology, vol. 20, no. 6, pp. 1605-1612, 2012.

[3] B. Xiao, Q. Hu, and Y. Zhang, "Fault-tolerant attitude control for flexible spacecraft without angular velocity magnitude measurement," Journal of Guidance, Control, and Dynamics, vol. 34, no. 5, pp. 1556-1561, 2011.

[4] Y. Zhao, W. Sun, and H. Gao, "Robust control synthesis for seat suspension systems with actuator saturation and time-varying input delay," Journal of Sound and Vibration, vol. 329, no. 21, pp. 4335-4353, 2010.

[5] L. Wu, X. Su, and P. Shi, "Sliding mode control with bounded $L_{2}$ gain performance of Markovian jump singular time-delay systems," Automatica, vol. 48, no. 8, pp. 1929-1933, 2012.

[6] Q. Hu, "Sliding mode maneuvering control and active vibration damping of three-axis stabilized flexible spacecraft with actuator dynamics," Nonlinear Dynamics, vol. 52, no. 3, pp. 227-248, 2008.

[7] G. Pujol, "Reliable $H_{\infty}$ control of a class of uncertain interconnected systems: an LMI approach," International Journal of Systems Science, vol. 40, no. 6, pp. 649-657, 2009. 
[8] Q. Zheng and F. Wu, "Nonlinear $H_{\infty}$ control designs with axisymmetric spacecraft control," Journal of Guidance, Control, and Dynamics, vol. 32, no. 3, pp. 850-859, 2009.

[9] W. Sun, H. Gao Sr., and O. Kaynak, "Finite frequency $H_{\infty}$ control for vehicle active suspension systems," IEEE Transactions on Control Systems Technology, vol. 19, no. 2, pp. 416-422, 2011.

[10] S. Yin, H. Luo, and S. Ding, "Real-time implementation of faulttolerant control systems with performance optimization," IEEE Transactions on Industrial Electronics, vol. 61, no. 5, pp. 24022411, 2013.

[11] M. V. Levskii, "Optimal control of reorientation of a spacecraft using free trajectory method," Cosmic Research, vol. 49, no. 2, pp. 131-149, 2011.

[12] E. M. Queen and L. Silverberg, "Optimal control of a rigid body with dissimilar actuators," Journal of Guidance, Control, and Dynamics, vol. 19, no. 3, pp. 738-740, 1996.

[13] S. Yin, S. X. Ding, A. H. A. Sari, and H. Hao, "Data-driven monitoring for stochastic systems and its application on batch process," International Journal of Systems Science, vol. 44, no. 7, pp. 1366-1376, 2013.

[14] S. Yin, S. X. Ding, A. Haghani, H. Hao, and P. Zhang, "A comparison study of basic data-driven fault diagnosis and process monitoring methods on the benchmark Tennessee Eastman process," Journal of Process Control, vol. 22, no. 9, pp. 1567-1581, 2012.

[15] S. Yin, X. Yang, and H. R. Karimi, "Data-driven adaptive observer for fault diagnosis," Mathematical Problems in Engineering, vol. 2012, Article ID 832836, 21 pages, 2012.

[16] S.-J. Dong, Y.-Z. Li, J. Wang, and J. Wang, "Fuzzy incremental control algorithm of loop heat pipe cooling system for spacecraft applications," Computers \& Mathematics with Applications, vol. 64, no. 5, pp. 877-886, 2012.

[17] Z. Gao, X. Shi, and S. X. Ding, "Fuzzy state/disturbance observer design for T-S fuzzy systems with application to sensor fault estimation," IEEE Transactions on Systems, Man, and Cybernetics $B$, vol. 38, no. 3, pp. 875-880, 2008.

[18] S. Udomsin, "Inverted pendulum control," KKU Engineering Journal, vol. 25, pp. 41-65, 2013.

[19] C.-H. Fang, Y.-S. Liu, S.-W. Kau, L. Hong, and C.-H. Lee, "A new LMI-based approach to relaxed quadratic stabilization of T-S fuzzy control systems," IEEE Transactions on Fuzzy Systems, vol. 14, no. 3, pp. 386-397, 2006.

[20] H. O. Wang, K. Tanaka, and M. F. Griffin, "An approach to fuzzy control of nonlinear systems: stability and design issues," IEEE Transactions on Fuzzy Systems, vol. 4, no. 1, pp. 14-23, 1996.

[21] H. O. Wang, K. Tanaka, and M. Griffin, "Parallel distributed compensation of nonlinear systems by Takagi-Sugeno fuzzy model," in Proceedings of the International Joint Conference of the 4th IEEE International Conference on Fuzzy Systems and the 2nd International Fuzzy Engineering Symposium, vol. 2, pp. 531538, March 1995.

[22] K. Tanaka, T. Ikeda, and H. O. Wang, "Fuzzy regulators and fuzzy observers: relaxed stability conditions and LMI-based designs," IEEE Transactions on Fuzzy Systems, vol. 6, no. 2, pp. 250-265, 1998.

[23] S. Boyd, L. EI Ghaoul, E. Feron, and V. Balakrishnan, Linear Matrix Inequalities in System and Control Theory, vol. 15 of Studies in Applied Mathematics, Society for Industrial Mathematics, 1994.

[24] P. Gahinet, A. Nemirovski, A. J. Laub, and M. Chilali, LMI Control Toolbox, vol. 1 of User's Guide Versión, Manuales de MATLAB, 1992. 


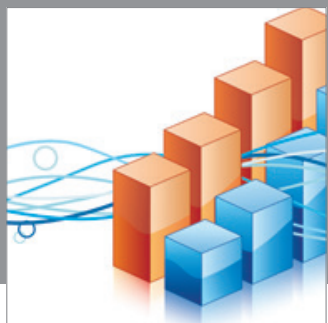

Advances in

Operations Research

mansans

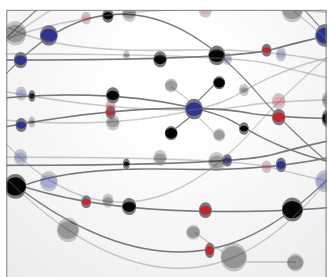

The Scientific World Journal
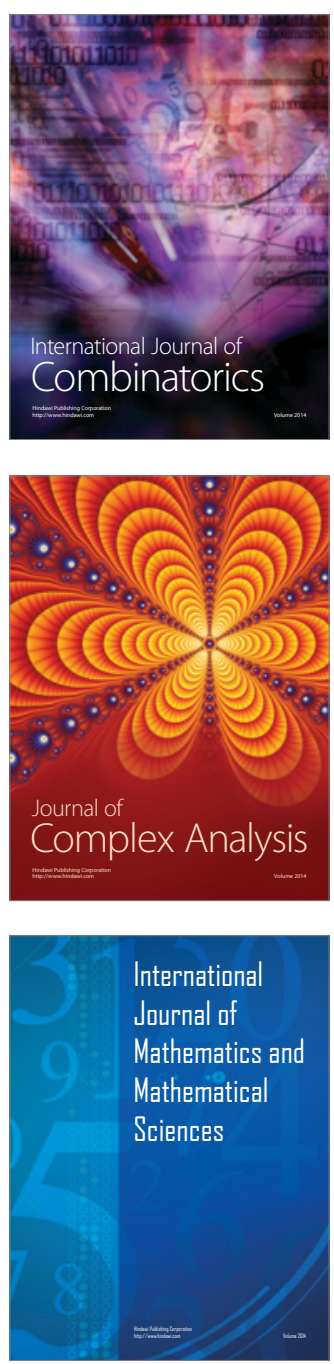
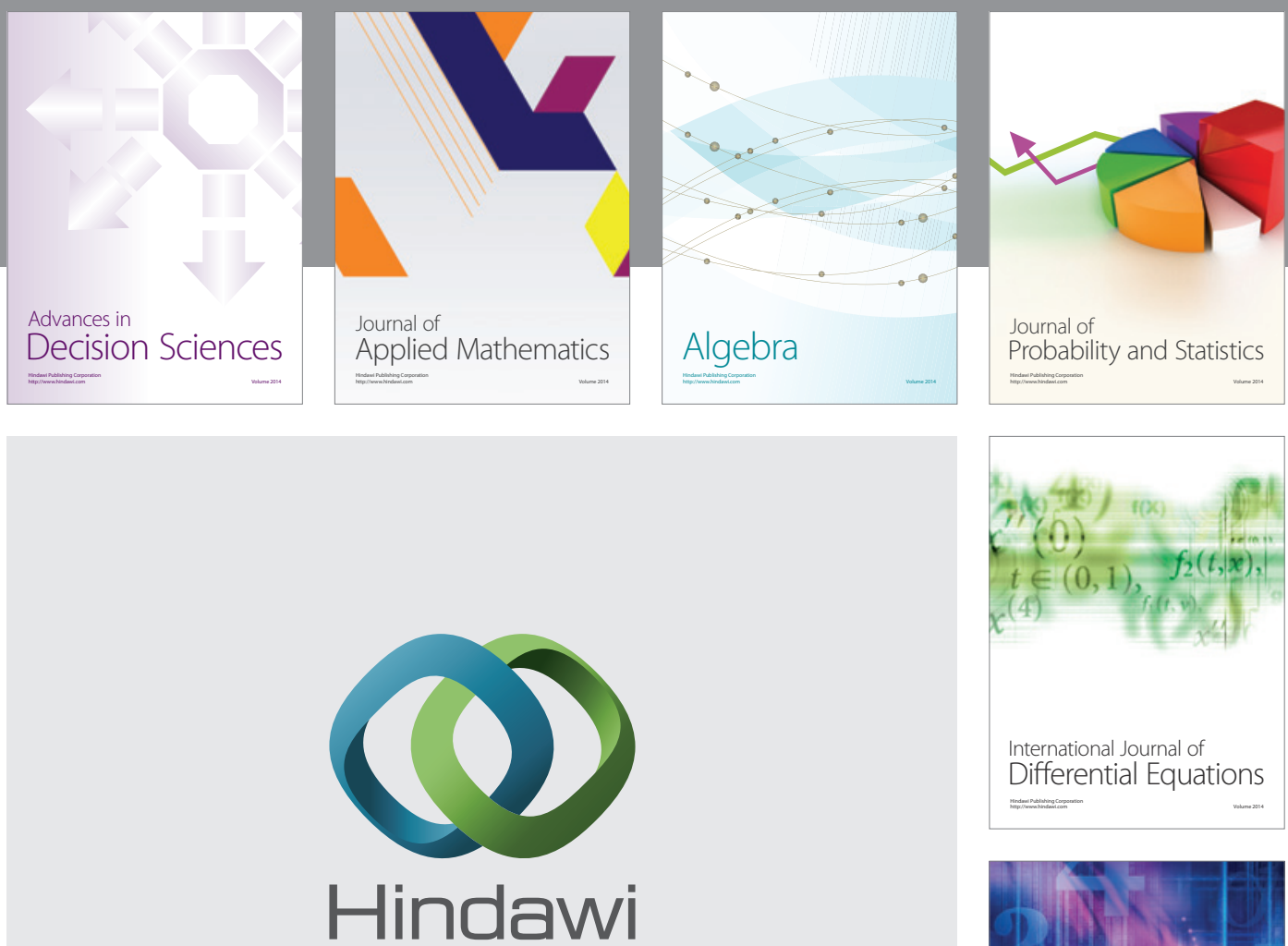

Submit your manuscripts at http://www.hindawi.com
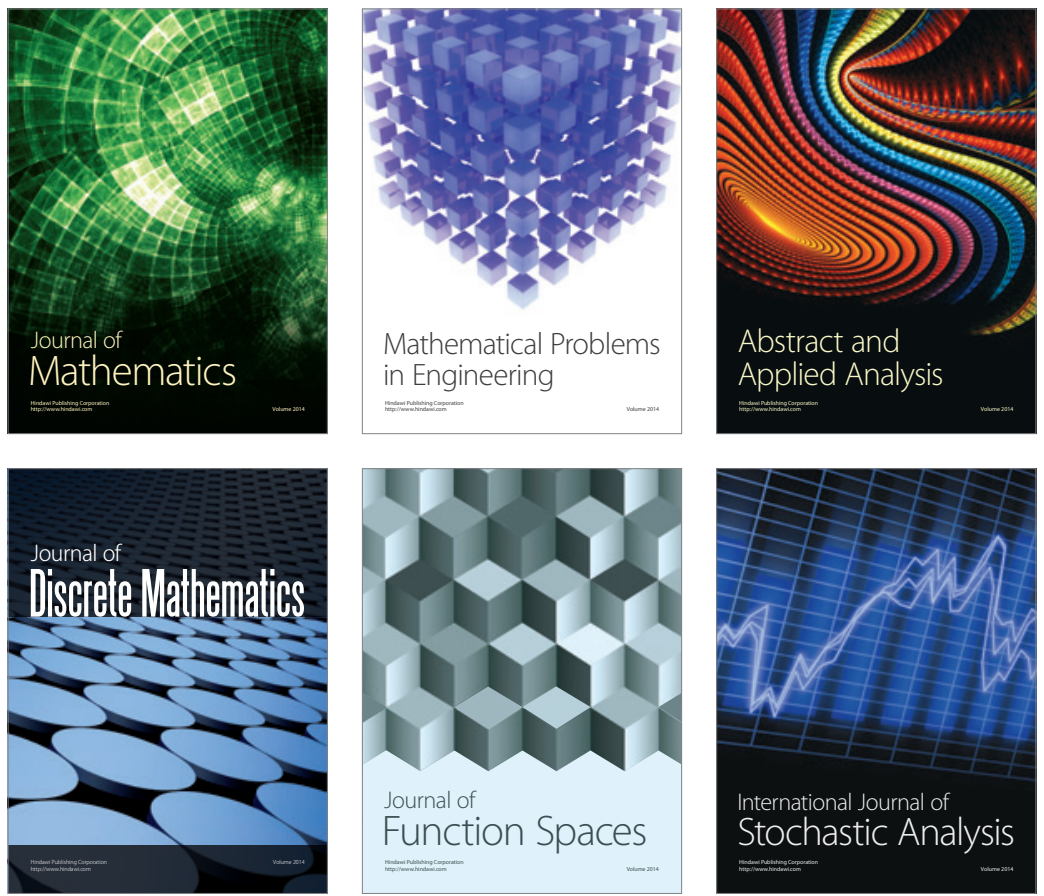

Journal of

Function Spaces

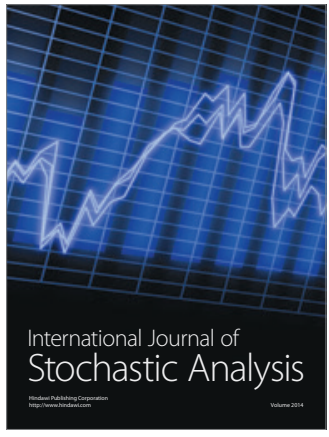

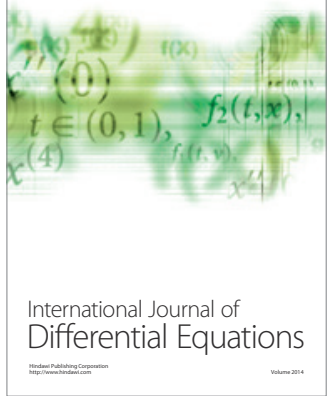
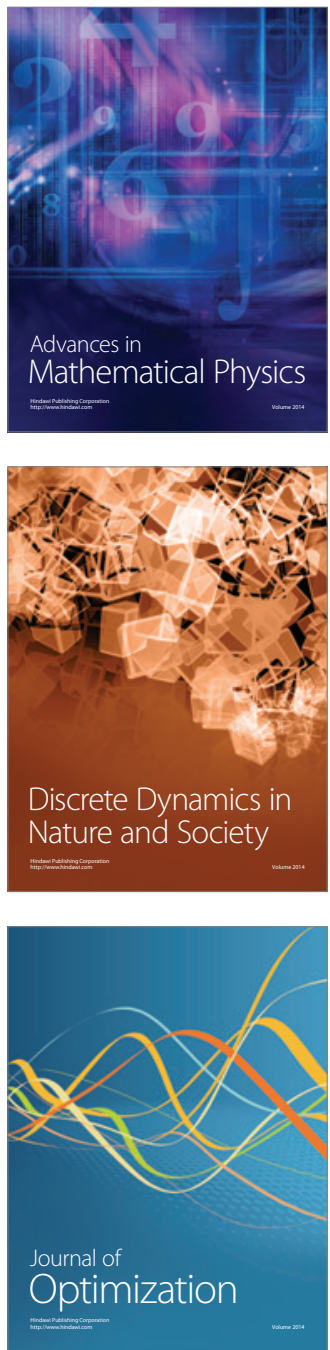